\title{
Caracterização epidemiológica e fatores de risco associados à leptospirose em ovinos deslanados do semiárido brasileiro ${ }^{1}$
}

\author{
Clebert J. Alves ${ }^{2}$, Jeferson F. Alcino ${ }^{2}$, Areano E.M. Farias ${ }^{2}$,Severino S.S. Higino ${ }^{2}$, Fabrine \\ A. Santos ${ }^{2}$, Sergio S. Azevedo ${ }^{2}$, Diego Figueiredo Costa $^{2}$ e Carolina S.A.B. Santos ${ }^{3}$
}

\begin{abstract}
Alves C.J., Alcino J.F., Farias A.E.M., Higino S.S.S., Santos F.A., Azevedo S.S., Costa D.F. \& Santos C.S.A.B. 2012. [Epidemiological characterization and risk factors associated with leptospirosis in the brazilian semiarid.] Caracterização epidemiológica e fatores de risco associados à leptospirose em ovinos deslanados do semiárido brasileiro. Pesquisa Veterinária Brasileira 32(6):523-528. Universidade Federal de Campina Grande, Centro de Saúde e Tecnologia Rural, Unidade Acadêmica de Medicina Veterinária, Av. Universitária s/nº, Patos, PB 58700-970, Brazil. E-mail: clebertja@uol.com.br

The aim of this investigation was to determine the herd-level and animal-level prevalence of leptospirosis in sheep from the semiarid of Paraíba State, Northeast Brazil, as well as to identify risk factors. Blood samples were collected from 1,275 sheep from 117 flocks in 19 counties in the Sertão mesoregion, Paraíba. For the serological diagnosis of leptospirosis the microscopic agglutination test (MAT) using 24 Leptospira spp. serovars as antigens was carried out. Of the 117 flocks studied $33(28.20 \%)$ presented at least one seropositive sheep, and of the 1,275 animals 69 (5.41\%) were seropositive with titers ranging from 100 to 3,200. Reactant serovars were Autumnalis (49.30\%), Andamana (27.53\%), Sentot (17.39\%), Whitcomb (4.34\%) and Australis (1.44\%). Herd size > 48 sheep (odds ratio $=2.26 ; 95 \% \mathrm{CI}=1.33-5.07 ; \mathrm{p}=0.021$ ) and participation in animal exhibits (odds ratio $=9.05$; $95 \% \mathrm{CI}=0.96-85.71 ; \mathrm{p}=0.055)$ were identified as risk factors. The need was suggested for studies on the isolation of the agent, characterization of its pathogenicity and its economic impact on sheep flocks of the region, and sanitary control in sheep agglomerations was recommended.
\end{abstract}

INDEX TERMS: Leptospira spp., leptospirosis, sheep, epidemiology, Sertão of the Paraíba.

RESUMO.- Este trabalho teve como objetivo determinar a prevalência de rebanhos ovinos positivos (focos) e de animais soropositivos para leptospirose na região semiárida do Estado da Paraíba, bem como identificar fatores de risco. Foram colhidas amostras de sangue de 1.275 animais procedentes de 117 rebanhos em 19 municípios da mesorregião do Sertão, Estado da Paraíba. Para o diagnóstico sorológico da leptospirose foi utilizado o teste de soroaglutinação microscópica (SAM), com 24 sorovares de

\footnotetext{
${ }^{1}$ Recebido em 10 de janeiro de 2012.

Aceito para publicação em 14 de fevereiro de 2012.

${ }^{2}$ Unidade Acadêmica de Medicina Veterinária, Centro de Saúde e Tecnologia Rural (CSTR), Universidade Federal de Campina Grande (UFCG), Av. Universitária s/n, Patos, PB 58700-970, Brasil. *Autor para correspondência: clebertia@uol.com.br

${ }^{3}$ Departmento de Medicina Veterinára Preventiva e Saúde Animal, Faculdade de Medicina Veterinária e Zootencia (FMVZ), Universidade de São Paulo (USP), Av. Prof. Dr. Orlando Marques de Paiva 87, Cidade Universitária, São Paulo, SP 05508-270, Brasil.
}

Leptospira spp. como antígenos. Um rebanho foi considerado positivo quando apresentou pelo menos um animal soropositivo. Das 117 propriedades utilizadas 33 (28,20\%) apresentaram pelo menos um animal soropositivo, e dos 1.275 animais $69(5,41 \%)$ foram soropositivos, com títulos variando de 100 a 3200 . 0 sorovares reagentes foram $\mathrm{Au}$ tumnalis (49,30\%), Andamana (27,53\%), Sentot $(17,39 \%)$, Whitcomb (4,34\%) e Australis (1,44\%). Possuir mais de 48 animais no rebanho (odds ratio $=2,26$; IC $95 \%=1,33-5,07$; $\mathrm{p}=0,021$ ) e participação em exposições (odds ratio $=9,05$; IC $95 \%=0,96-85,71 ; p=0,055$ ) foram identificados como fatores de risco. Sugere-se a necessidade de estudos acerca do isolamento do agente, da caracterização de sua patogenicidade e do seu impacto econômico nos rebanhos ovinos da região, bem como recomenda-se maior controle sanitário nas aglomerações de animais.

TERMOS DE INDEXAÇÃO: Leptospira spp., leptospirose, ovinos, epidemiologia, Sertão da Paraíba. 


\section{INTRODUÇÃo}

A ovinocultura brasileira destaca-se no cenário nacional por apresentar um grande potencial de crescimento, tendo-se observado, nos últimos anos, uma evolução significativa no rebanho nacional, contando hoje com um efetivo de ovinos que chega a mais de 16 milhões de cabeças (Brasil 2009). Essa exploração apresenta inúmeras vantagens, como necessidade de menor área de criação, menor consumo de alimentos, facilidade de manejo e grande diversidade de produção de carne e couro de boa qualidade, servindo como alternativa de renda. Contudo, deficiências sanitárias envolvidas no processo evolutivo da ovinocultura brasileira necessitam de resolução, tais como os prejuízos provocados pelas perdas reprodutivas, associados à necessidade de reposição dos animais (Fernandes 2009).

A leptospirose está mundialmente distribuída, mas sua incidência tem forte associação com períodos de alta pluviosidade (Alves et al. 1996, Vanasco et al., 2008) e sob condições favoráveis e na presença de hospedeiros adequados, as leptospiras podem persistir por semanas a meses no ambiente (Faine et al. 1999, Adler \& De La Pena 2010).

A infecção nos ovinos pode se manifestar sob a forma aguda, crônica ou inaparente. Os quadros clínicos mais característicos são de septicemia, hemorragia e nefrite, seguida por icterícia, hemoglobinúria, mastite sanguinolenta, retorno ao cio, abortamento nas ovelhas e anemia hemolítica nos cordeiros com morte na primeira semana de vida. No entanto, a forma inaparente é muito mais frequente e desperta pouca atenção dos pesquisadores devido à dificuldade no diagnóstico. Por outro lado, do ponto de vista epidemiológico, é uma forma muito importante, uma vez que a introdução de animais com infecção inaparente pode garantir a persistência do agente nos rebanhos acometidos (Ciceroni et al. 2000).

Entre os fatores envolvidos na infecção por Leptospira spp. nas criações de ovinos, a criação consorciada com bo- vinos tem sido apontada como fator principal, onde os ovinos adquirem a infecção pela urina e bebedouros coletivos (Fernandes 2009, Escócio et al. 2010).

Estudos recentes conduzidos em caprinos e ovinos no semiárido nordestino apontaram predominância de reações para o sorovar Autumnalis. Araújo Neto et al. (2008) examinaram soros de 366 caprinos do Rio Grande do Norte e referiram que o sorovar mais frequente foi o Autumnalis, com $73,6 \%$ das reações positivas. Araújo Neto (2005) utilizou 100 ovelhas abatidas no matadouro público de Patos, $\mathrm{PB}$, para o isolamento da bactéria a partir do trato genital, realizando paralelamente a sorologia, obtendo $9 \%$ de soropositividade para a doença, e $44,4 \%$ de frequência para o sorovar Autumnalis. Higino et al. (2010) realizaram a sorologia de 80 ovinos e obteve $7,5 \%$ de positividade, com $83,3 \%$ de frequência para o sorovar Autumnalis.

0 presente trabalho teve como objetivo determinar a prevalência de propriedades positivas (focos) e de animais soropositivos para a infecção por Leptospira spp., bem como identificar possíveis fatores de risco em ovinos deslanados no semiárido da Paraíba.

\section{MATERIAL E MÉTODOS}

O Estado da Paraíba é dividido geograficamente em quatro mesorregiões (Sertão, Borborema, Agreste e Mata Paraibana) e 23 microrregiões. A mesorregião do Sertão Paraibano possui como principal atividade a pecuária extensiva, assumindo destaque a criação de ovinos. 0 rebanho ovino brasileiro é de mais de $16 \mathrm{mi}-$ lhões de cabeças, das quais 56,90\% (9.566.968) estão na região Nordeste. Desse efetivo de ovinos nordestinos, 4,54\% (434.225) são encontrados na Paraíba distribuídos em aproximadamente 19.744 estabelecimentos (Brasil 2009). No presente trabalho foram utilizados ovinos deslanados adultos provenientes da mesorregião do Sertão (Figura 1).

A amostragem foi delineada para a determinação da prevalência de propriedades positivas e de animais soropositivos, de maneira que foi conduzida em dois estágios. Inicialmente, as propriedades foram aleatoriamente selecionadas. 0 número de

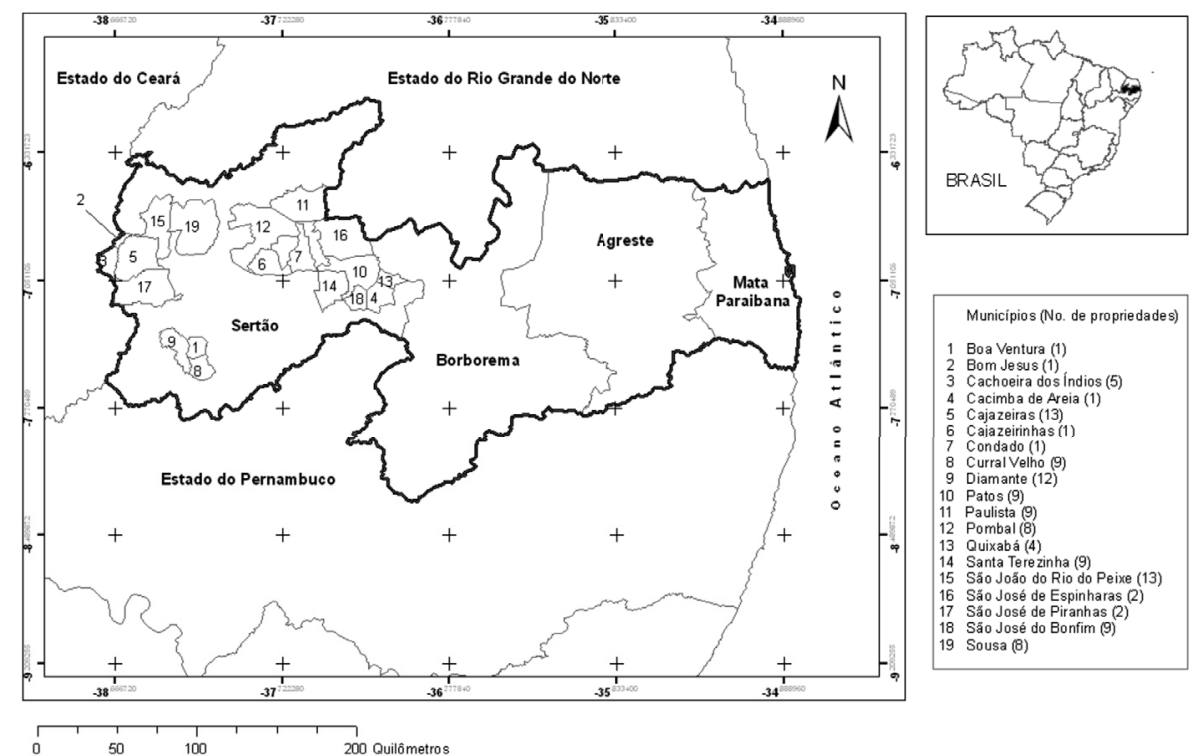

Fig.1. Mesorregiões do Estado da Paraíba demonstrando os municípios e respectivos números de propriedades rurais utilizadas, na mesorregião do Sertão paraibano. 
propriedades a serem amostradas foi calculado com o programa Epiinfo versão 6.04 (Dean 1994), com o emprego dos seguintes parâmetros: prevalência esperada de 50\% (valor adotado para maximizar a amostra), nível de confiança de 95\% e erro absoluto de 10\% (Thrusfield 1995). Na mesorregião do Sertão o total de propriedades criadoras de ovinos é de 7.087, resultando em uma amostra de 96 propriedades. Por motivo de segurança, foram visitadas 117 propriedades.

O número de ovinos a serem selecionados foi determinado individualmente por propriedade com o objetivo de detectar a presença da infecção, utilizando a seguinte fórmula (Thrusfield 1995):

$$
n=\left[1-(1-p)^{\frac{1}{d}}\right] \times\left(\mathrm{N}-\frac{\mathrm{d}}{2}\right)+1
$$

onde:

$n=$ tamanho da amostra;

$p=$ probabilidade de detectar pelo menos um animal soropositivo;

$N=$ tamanho do rebanho;

$d-=$ número de animais soropositivos no rebanho.

A probabilidade de detecção de pelo menos um animal soropositivo foi de $95 \%(p=0,95)$, e o número de animais soropositivos por rebanho (d) foi calculado assumindo prevalência intra-rebanho de $10 \%$.

No total, foram utilizados 1.275 animais procedentes de 117 propriedades de 19 municípios da mesorregião do Sertão paraibano (Fig.1).

0 trabalho de campo foi desenvolvido no período de julho de 2009 a julho de 2010. As atividades de campo incluíram a colheita de sangue, aplicação do questionário epidemiológico, e envio para o Laboratório de Doenças Transmissíveis (LDT) do Centro de Saúde e Tecnologia Rural (CSTR) da Universidade Federal de Campina Grande (UFCG), em Patos, PB. As amostras de sangue foram colhidas de ovinos deslanados adultos, em volumes de $8 \mathrm{ml}$, pela punção da veia jugular com agulha descartável e tubo a vácuo (sem anticoagulante) com capacidade de 8,5 mL. Após o dessoramento, o soro foi transferido para microtubos e congelados até a realização da prova sorológica.

0 diagnóstico sorológico de leptospirose foi realizado com a técnica de Soroaglutinação Microscópica (SAM), de acordo com Galton et al. (1965) e Cole et al. (1973), utilizando uma coleção de antígenos vivos que incluiu os sorovares Castellonis, Javanica, Tarassovi, Whitcombi, Australis, Autumnalis, Bataviae, Bratislava, Canicola, Copenhageni, Grippotyphosa, Hardjo (estirpes Hardjoprajitno e Hardjobovis), Hebdomadis, Pomona, Icterohaemorrhagiae, Sentot, Wolffi, Pyrogenes, Butembo, Cynopteri, Panama, Shermani, Andamana e Patoc. Os soros foram triados na diluição de 1:100, e aqueles que apresentaram $50 \%$ ou mais de aglutinação foram titulados pelo exame de uma série de diluições geométricas de razão dois. 0 título do soro foi a recíproca da maior diluição que apresentou resultado positivo. Os antígenos foram examinados ao microscópio de campo escuro, previamente aos testes, a fim de verificar a mobilidade e a presença de auto-aglutinação ou de contaminantes.

Para a análise de possíveis fatores de risco associados com a condição de propriedade positiva para a infecção por Leptospira spp. foram utilizados os dados colhidos nos questionários epidemiológicos. Uma propriedade foi considerada positiva quando apresentou pelo menos um animal soropositivo. A análise de fatores de risco foi conduzida em duas etapas: análise univariada e análise multivariada. $\mathrm{Na}$ análise univariada, cada variável independente foi cruzada com a variável dependente, e aquelas que apresentaram valor de $\mathrm{p} \leq 0,15$ pelo teste de qui-quadrado (Zar 1999) foram selecionadas para a análise multivariada, utilizando-se a regressão logística múltipla (Hosmer \& Lemeshow 2000). 0 nível de significância adotado na análise múltipla foi de $5 \%$. Todas as análises foram realizadas com o programa SPSS 20.0 for Windows.

\section{RESULTADOS E DISCUSSÃO}

Em 33 (28,20\%) propriedades, distribuídas em 13 municípios, houve pelo menos um animal soropositivo para um dos sorovares de Leptospira spp. utilizados, e dos 1.275 animais $69(5,41 \%)$ foram soropositivos, o que sugere que a infecção encontra-se disseminada nos rebanhos ovinos da região.

A prevalência de propriedades positivas encontrada no presente trabalho $(28,20 \%)$ foi menor do que a observada por Herrmann et al. (2004), que em estudo realizado no Rio Grande do Sul, detectou um total de 113 (83,09\%) propriedades positivas entre 136 utilizadas, com pelo menos um animal reagente. Essa disparidade entre os resultados encontrados provavelmente é justificada pelo fato do Estado do Rio Grande do Sul apresentar altos índices pluviométricos, diferentes sistemas de criação e maior quantidade de áreas irrigadas, facilitando a disseminação de Leptospira spp.

Com relação ao título de anticorpos, 27 (39,1\%) animais apresentaram título 200; 24 (34,8\%) título 100; 10 $(14,5 \%)$ título 400 ; quatro $(5,8 \%)$ título 1600 ; três $(4,3 \%)$ título 800; e um $(1,4 \%$ ) título 3200 (Quadro 1). Foram observadas reações positivas para os seguintes sorovares: $34(49,30 \%)$ animais soropositivos para Autumnalis; 19 $(27,53 \%)$ para Andamana; $12(17,39 \%)$ para Sentot; três $(4,34 \%)$ para Whitcombi; e um $(1,44 \%)$ para Australis.

0 sorovar mais frequente no presente trabalho foi 0 Autumnalis. Este sorovar possui como principais reservatórios os roedores (Faine et al. 1999), indicando a impor-

Quadro 1. Sorovares de Leptospira spp. mais prevalentes e respectivos títulos em ovinos deslanados soropositivos na mesorregião do Sertão paraibano, no período de julho de 2009 a julho de 2010

\begin{tabular}{lccccccc}
\hline \multicolumn{7}{c}{ Sorovares } & \multicolumn{9}{c}{ Títulos } & \multicolumn{2}{c}{ Total (\%) } \\
\cline { 2 - 7 } & 100 & 200 & 400 & 800 & 1600 & 3200 & \\
\hline Andamana & 3 & 7 & 5 & 2 & 2 & - & $19(27,53)$ \\
Australis & - & - & 1 & - & - & - & $1(1,44)$ \\
Autummalis & 15 & 13 & 3 & - & 2 & 1 & $34(49,30)$ \\
Sentot & 4 & 7 & 1 & - & - & - & $12(17,39)$ \\
Whitcombi & 2 & - & - & 1 & - & - & $3(4,34)$ \\
Total (\%) & $24(34,71)$ & $27(39,1)$ & $10(14,49)$ & $3(4,34)$ & $4(5,7)$ & $1(1,4)$ & $69(100)$
\end{tabular}


Quadro 2. Análise univariada para fatores de risco associados á condição de foco de infecção por Leptospira spp. em ovinos deslanados na mesorregião do Sertão paraibano, no período de julho de 2009 a julho de 2010

\begin{tabular}{|c|c|c|c|c|c|}
\hline Variável & $\begin{array}{c}\text { № total de } \\
\text { propriedades }\end{array}$ & $\begin{array}{l}\text { Propriedades } \\
\text { positivas (\%) }\end{array}$ & Odds ratio & IC 95\% & $\mathrm{p}$ \\
\hline \multicolumn{6}{|l|}{ Tipo de criação } \\
\hline Intensiva & 6 & $5(83,3)$ & 11,61 & $1,22-275,07$ & $0,015^{*}$ \\
\hline Extensiva/semi-intensiva & 93 & $28(30,1)$ & 1 & & \\
\hline \multicolumn{6}{|l|}{ Finalidade da criação } \\
\hline Cria & 28 & $6(21,4)$ & 1 & & \\
\hline Recria/engorda & 45 & $16(35,6)$ & 2,02 & $0,61-6,96$ & 0,309 \\
\hline Reprodução & 9 & $2(22,2)$ & 1,05 & $0,11-8,22$ & 1,000 \\
\hline Subsistência & 15 & $8(53,3)$ & 4,19 & $0,9-20,71$ & $0,046^{*}$ \\
\hline \multicolumn{6}{|l|}{ Tipo de exploração } \\
\hline Corte & 80 & $25(31,3)$ & 1 & & \\
\hline Leite & 3 & $1(33,3)$ & 1,10 & $0,02-22,03$ & 1,000 \\
\hline Outros & 16 & $7(43,8)$ & 1,71 & $0,5-5,79$ & 0,498 \\
\hline \multicolumn{6}{|l|}{ Criação tecnificada } \\
\hline Não & 82 & $25(30,5)$ & 1 & & \\
\hline Sim & 16 & $7(43,8)$ & 1,77 & $0,52-5,99$ & 0,457 \\
\hline \multicolumn{6}{|c|}{ Principal atividade da propriedade } \\
\hline Não & 76 & $25(32,9)$ & 1 & & \\
\hline Sim & 23 & $8(34,8)$ & 1,09 & $0,36-3,21$ & 0,933 \\
\hline \multicolumn{6}{|l|}{ Tamanho do rebanho } \\
\hline Até 19 & 29 & $6(20,7)$ & 1 & & \\
\hline $20-48$ & 48 & $13(27,1)$ & 1,42 & $0,42-4,95$ & 0,720 \\
\hline$>48$ & 23 & $14(60,9)$ & 5,96 & $1,5-24,97$ & $0,007 *$ \\
\hline \multicolumn{6}{|l|}{ Contato com outros animais } \\
\hline Não & 39 & $14(35,9)$ & 1,24 & $0,48-3,20$ & 0,780 \\
\hline Sim & 58 & $18(31,0)$ & 1 & & \\
\hline \multicolumn{6}{|l|}{ Pastagem nativa } \\
\hline Não & 2 & $1(50,0)$ & 2,06 & $0,03-164,31$ & 1,000 \\
\hline Sim & 98 & $32(32,7)$ & 1 & & \\
\hline \multicolumn{6}{|l|}{ Suplementação } \\
\hline Não & 50 & $18(36,0)$ & 1,05 & $0,41-2,69$ & 0,916 \\
\hline Sim & 43 & $15(34,9)$ & 1 & & \\
\hline \multicolumn{6}{|l|}{ Aquisição frequente de animais } \\
\hline Não & 36 & $11(30,6)$ & 1 & & \\
\hline Sim & 61 & $22(36,1)$ & 1,28 & $0,49-3,4$ & 0,740 \\
\hline \multicolumn{6}{|l|}{ Participação em exposições } \\
\hline Não & 80 & $25(31,3)$ & 1 & & \\
\hline Sim & 7 & $6(85,7)$ & 13,20 & $1,43-306,82$ & $0,007 *$ \\
\hline \multicolumn{6}{|l|}{ Vacinações } \\
\hline Não & 72 & $23(31,9)$ & 1 & & \\
\hline Sim & 26 & $10(38,5)$ & 1,33 & $0,47-3,73$ & 0,718 \\
\hline \multicolumn{6}{|l|}{ Vermifugações } \\
\hline Não & 12 & $2(16,7)$ & 1 & & \\
\hline Sim & 88 & $31(35,2)$ & 2,72 & $0,51-19,25$ & 0,327 \\
\hline \multicolumn{6}{|l|}{ Presença de abortos } \\
\hline Não & 59 & $21(35,6)$ & 1,21 & $0,45-3,23$ & 0,851 \\
\hline Sim & 35 & $11(31,4)$ & 1 & & \\
\hline \multicolumn{6}{|l|}{ Nascimento de crias mortas } \\
\hline Não & 65 & $23(35,4)$ & 1,3 & $0,45-3,84$ & 0,772 \\
\hline Sim & 27 & $8(29,6)$ & 1 & & \\
\hline Morte dos cordeiros nas prin & & & & & \\
\hline Não & 65 & $21(32,3)$ & 1 & & \\
\hline Sim & 24 & $10(41,7)$ & 1,5 & $0,51-4,36$ & 0,567 \\
\hline Morte após desmame & 74 & $24(32,4)$ & & & \\
\hline Não & 15 & $5(33,3)$ & 1 & & \\
\hline Sim & & & 1,04 & $0,27-3,84$ & 1,000 \\
\hline Comportamento homossexua & & & & & \\
\hline Não & 42 & $14(33,3)$ & 1 & & \\
\hline Sim & 48 & $19(39,6)$ & 1,31 & $0,51-3,4$ & 0,693 \\
\hline Presença de plantas toxica & & & & & \\
\hline Não & 34 & $14(41,2)$ & 1,67 & $0,64-4,41$ & 0,354 \\
\hline Sim & 61 & $18(29,5)$ & 1 & & \\
\hline
\end{tabular}

*Variáveis usadas na regressão logística múltipla. 
tância desses animais como prováveis fontes de infecção para os ovinos, bem como a necessidade de intensificação do controle dos mesmos. Silva et al. (2007), utilizando rins de ovinos abatidos em Pelotas, RS, obtiveram um isolado de Leptospira noguchii que foi identificado como pertencente ao sorogrupo Autumnalis. Estudos conduzidos em caprinos e ovinos no semiárido nordestino, que apontaram predominância de reações para o sorovar Autumnalis (Araújo Neto 2005, Araújo Neto et al. 2008, Higino et al. 2010), bem como os resultados do presente trabalho, levantam a hipótese dos ovinos como fontes de infecção do sorovar Autumnalis na região e reforçam o risco de transmissão para os seres humanos e para outras espécies. Saravanan et al. (2000), na Índia, isolaram o sorovar Autumnalis de um paciente com leptospirose confirmada clinicamente, na área suburbana de Madras.

O sorovar Andamana, segundo mais frequente, pertence à espécie $L$. biflexa, é apatogênico e de vida livre, entretanto, é utilizado como marcador sorológico, pois costuma reagir precocemente, e apresenta reações cruzadas com sorovares patogênicos (Aguiar et al. 2010).

No Brasil, são poucos os relatos de isolamento de leptospiras e seu papel na etiologia da leptospirose. Na Paraíba, Higino et al. (2010) isolaram leptospiras do trato reprodutivo de ovinos abatidos sem, contudo, realização de tipificação dos isolados, o que reforça a necessidade da condução de estudos visando o isolamento do agente na região e a investigação de sua patogenicidade.

No tocante à análise de fatores de risco, as variáveis mais associadas $(p \leq 0,15)$ à ocorrência de focos na análise univariada foram: tipo de criação $(\mathrm{p}=0,015)$, finalidade da criação $(\mathrm{p}=0,046)$, tamanho do rebanho $(\mathrm{p}=0,007)$ e participação em exposições ( $p=0,007$ ) (Quadro 2). Na análise multivariada, as variáveis apontadas como fatores de risco foram: tamanho do rebanho $>48$ animais (odds ratio $=2,26$; IC $95 \%=1,33-5,07 ; p=0,021$ ) e participação em exposições (odds ratio $=9,05 ;$ IC 95\% $=0,96-85,71 ; \mathrm{p}=0,055$ ) (Quadro 3).

O tamanho do rebanho > 48 animais e participação em exposições, apontados como fatores de risco, refletem as condições de aglomerações de animais, que são sabidamente favoráveis à disseminação de doenças para os susceptíveis dentro das populações, sendo as chances cada vez maiores à medida que o número de animais e o tempo de exposição aumentam nestas populações (Thrusfield 1995). Faine (1982) referiu que dentro desta condição a sanidade do rebanho é um fator critico para a infecção por Leptospira spp., onde animais presentes nessas aglomerações são mais expostos ao contato com fômites, urina de animais portadores assintomáticos, secreções vaginais e abortos de animais acometidos. Embora apresentando valor de p no

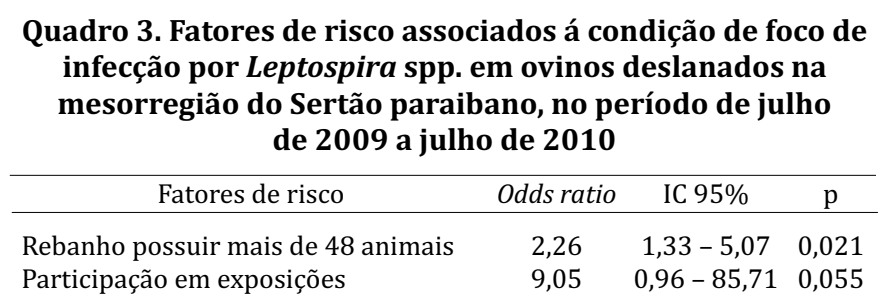

limite da significância estatística $(\mathrm{p}=0,055)$, foi conveniente considerar a variável participação em exposições como fator de risco, tendo em vista a plausibilidade biológica.

Outras variáveis, embora não terem sido apontadas como fatores de risco na análise multivariada, merecem destaque. A finalidade de criação de subsistência reflete as condições mais rudimentares de tais propriedades, bem como a ausência de assistência veterinária frequente, onde os proprietários geralmente só consideraram a visita de um veterinário quando se observa grandes perdas na criação. Lilembaum et al. (2008) observaram que rebanhos caprinos que não tinham assistência veterinária apresentaram maior chance de serem focos de leptospirose comparados com aqueles que contavam com tal assistência.

\section{CONCLUSÕES}

Constatou-se que $28,20 \%$ das propriedades tiveram animais reagentes e que $5,41 \%$ dos animais foram soropositivos, bem como o sorovar Autumnalis foi o mais frequente, o que sugere a necessidade de estudos visando o isolamento do agente na região e a caracterização de sua patogenicidade, bem como o seu impacto econômico nos rebanhos ovinos da região.

Os fatores de risco apontados foram o tamanho do rebanho e, portanto, não passível de intervenção, e a participação em exposições, o que sugere um maior controle sanitário nas aglomerações de animais.

\section{REFERÊNCIAS}

Adler B. \& De La Pena M.A. 2010. Leptospira and leptospirosis. Vet. Microbiol. 140(3/4):287-296.

Aguiar D.M., Cavalcante G.T., Vasconcellos S.A., Souza G.O., Labruna M.B., Camargo L.M.A. \& Gennari S.M. 2010. Anticorpos anti-Leptospira spp. em ovinos do Município de Monte Negro, Estado de Rondônia. Arqs Inst. Bioógico, São Paulo, 77(3):529-532.

Alves C.J., Vasconcellos S.A., Camargo C.R.A. \& Morais Z.M. 1996. Influência dos fatores ambientais sobre a proporção de caprinos soro-reatores para a leptospirose em cinco centros de criação do Estado da Paraíba, Brasil. Arqs Inst. Biológico, São Paulo, 63(2):11-18.

Araújo Neto J.O. 2005. Isolamento de Leptospira spp. a partir do trato genital de ovelhas abatidas no Matadouro Público de Patos/PB, Estado de Paraíba, Brasil. Monografia de Conclusão de Curso de Graduação em Medicina Veterinária, Universidade Federal de Campina Grande, Patos, PB. 58p.

Araújo Neto J.O., Silva M.L.C.R., Azevedo S.S., Batista C.S.A., Gomes A.A.B., Alves C.J., Lima F.S. \& Alves F.A.L. 2008. Soroprevalência e fatores de risco associados à infecção por Leptospira spp. em rebanhos caprinos da microrregião do Seridó Oriental, Rio Grande do Norte, Brasil. Anais Encontro Nacional de Diagnóstico Veterinário, Campo Grande, MS, p.201202.

Brasil 2009. Pesquisa da pecuária municipal. Sistema Instituto Brasileiro de Geografia e Estatística (IBGE) de recuperação automática (SIDRA). Disponível em <http://www.sidra.ibge.gov.br/bda/tabela/listabl. asp?c=73\&z=t\&o=20> Acesso 30 nov. 2011.

Ciceroni L., Lombardo D., Pinto A., Ciarrocchi S. \& Simeoni J. 2000. Prevalence of antibodies to Leptospira serovars in sheep and goats in Alto Adige-South Tyrol. J. Vet. Med. 47(5):217-223.

Cole J.R., Sulzer C.R. \& Pulssely P.R. 1973. Improved microtechnique for the leptospiral microscopic agglutination. Appl. Microbiol. 28(5):976-980.

Dean A.G. 1994. Epiinfo version 6: a word-processing, database, and statistic program for public health on IBM-compatible microcomputers. Center for Diseases Control and Prevention, Atlanta. 601p. 
Escócio C., Genovez M.E., Castro V., Piatti R.M., Gabriel F.H.L., Chiebao D.P., Azevedo S.S., Vieira S.R. \& Chiba M. 2010. Influência das condições ambientais na transmissão da leptospirose entre criações de ovinos e bovinos da região de Sorocaba, SP. Arqs Inst. Biológico, São Paulo, 77(3):371-379.

Faine S. 1982. Guidelines for the control of leptospirosis. World Health Organization, Genebra. 171p.

Faine S., Adler B., Bolin C. \& Perolat P. 1999. Leptospira and leptospirosis. $2^{\text {nd }}$ ed. MediSci, Melbourne. 272p.

Fernandes C.E. 2009. Papel do ovino na cadeia epidemiológica da leptospirose pela Leptospira spp. sorovar Hardjo: fatores de risco que envolvem a infecção e transmissão entre ovinos e bovinos. Dissertação de Mestrado em Sanidade Animal, Segurança Alimentar e o Ambiente, Instituto Biológico de São Paulo, SP. 101p.

Galton M.M., Sulzer C.R., Santa Rosa C.A. \& Fields M.J. 1965. Application of a microtechnique to the agglutination test for leptospiral antibodies. Appl. Microbiol. 13:81-85.

Herrmann G.P., Lage A.P. \& Moreira E.C. 2004. Soroprevalência de aglutininas anti-Leptospira spp. em ovinos nas Mesorregiões Sudeste e Sudoeste do Estado Rio Grande do Sul, Brasil. Ciência Rural 34(2):443448.

Higino S.S.S., Azevedo S.S., Alves C.J., Figueiredo S.M., Silva M.L.C.R. \& Batis- ta C.S.A. 2010. Frequência de leptospirose em ovinos abatidos no Município de Patos, Paraíba. Arqs Inst. Biológico, São Paulo, 77(3):525-527.

Hosmer D.W. \& Lemeshow S. 2000. Applied logistic regression. John Wiley and Sons, New York. 375p.

Lilembaum W., Varges R., Medeiros L., Cordeiro A.G., Cavalcanti A., Souza G.N., Richtzenhain L. \& Vasconcellos S.A. 2008. Risk factors associated with leptospirosis in dairy goats under tropical conditions in Brazil. Res. Vet. Sci. 84:4-17.

Saravanan R., Rajendran P., Thyagarajan S.P., Smythe L.D., Norris M.A., Symonds M.L. \& Dohnt M.F. 2000. Leptospira autumnalis isolated from a human case from Avadi, India, and the serovar's predominance in local rat and bandicoot populations. Ann. Trop. Med. Parasitol. 94(5):503-506.

Silva E.F., Brod C.S., Cerqueira G.M., Bourscheidt D., Seyffert N., Queiroz A., Santos C.S., Ko A.I. \& Dellagostin O.A. 2007. Isolation of Leptospira noguchii from sheep. Vet. Microbiol. 121:144-149.

Thrusfield M. 1995. Veterinary epidemiology. $2^{\text {nd }}$ ed. Blackwell Science, Cambridge. $479 \mathrm{p}$.

Vanasco N.B., Schmeling M.F., Lottersberger J., Costa F., Ko A.I. \& Tarabla H.D. 2008. Clinical characteristics and risk factors of human leptospirosis in Argentina (1999-2005). Acta Trop. 107:255-258.

Zar J.H. 1999. Biostatistical Analysis. $4^{\text {th }}$ ed. Prentice Hall, Upper Saddle River. 663p. 